\title{
La difusión de la investigación científica en Perú: implicaciones en la educación superior
}

\author{
Israel Barrutia Barreto \\ Universidad Científica del Sur. Lima, Perú.
}

\author{
Erika Raquel Acosta Roa \\ Innova Scientific. Lima, Perú.
}

Manuel Quipuscoa Silvestre

Universidad Nacional de Trujillo. Trujillo, Perú.

\author{
Herbert Victor Huaranga Rivera \\ Universidad Nacional Intercultural de la Selva Central Juan Santos Atahualpa. Chanchamayo, Perú.
}

ORIGINAL

\begin{abstract}
Resumen
Objetivo. Este estudio se plantea explorar la producción de los investigadores peruanos, analizando el estado de sus publicaciones y su impacto en la educación superior. Método. Se analizará la actuación de las publicaciones peruanas en los bancos de información de Scimago Journal \& Country Rank, a través del número de citas, el índice $\mathrm{H}$ de Hirsh y el Factor de Impacto, conjuntamente con los datos aportados por el Reglamento de calificación, clasificación y registro de los investigadores RENACYT. El procesamiento de la información se realizó mediante tablas, gráficos y el análisis estadístico descriptivo de la información recopilada. Resultados. Los resultados evidencian que las publicaciones del país han mostrado una tendencia favorable al crecimiento, sin embargo al compararse con otros países de la región su actuación es moderada y lenta, situación que influye notoriamente en los parámetros establecidos en el Reglamento de calificación, clasificación y registro de los investigadores RENACYT. Conclusiones Este panorama impacta sobre la redefinición de la investigación científica en la educación superior, ya que a través de la aplicación del Reglamento de calificación, clasificación y registro de los investigadores RENACYT se espera elevar el nivel del investigador, introduciendo cambios que afectan de manera directa a los docente quienes deben cumplir una serie de estrictos requisitos para poder ser catalogados como investigadores.
\end{abstract}

Palabras clave:

Difusión de la investigación científica. Docente. Educación superior. Investigación científica.

\section{The dissemination of scientific research in Peru: Implications in higher education}

\begin{abstract}
Objective. This study aims to explore the production of Peruvian researchers, analyzing the status of their publications and their impact on higher education. Method. The performance of Peruvian publications will be analyzed in the information banks of Scimago Journal \& Country Rank Country Rank, through the number of citations, the Hirsh $\mathrm{H}$ index and the Impact Factor, together with the data provided by the Qualification Regulation. , classification and registration of RENACYT researchers. The information was processed using tables, graphs and the descriptive statistical analysis of the information collected. Results. The results show that the country's publications have shown a favorable growth trend, however, when compared to other countries in the region, their performance is moderate and slow, a situation that has a marked influence on the parameters established in the Regulations for qualification, classification and registration of the RENACYT researchers. Conclusions. This panorama impacts on the redefinition of scientific research in higher education, since through the application of the Regulation of qualification, classification and registration of RENACYT researchers it is expected to raise the level of the researcher, introducing changes that affect directly to teachers who must meet a series of strict requirements to be cataloged as researchers.
\end{abstract}




\section{Introdución}

La sociedad está conformada por varias de instituciones, que muchas veces funcionan como un sistema que se interrelaciona entre sí, para muchos autores la sociedad actúa como un todo que se articula en la que los fenómenos se encuentran orgánicamente vinculados unos a otros, dependiendo unos de otros y a la vez se condicionan entre sí (Maturana y Varela, 1994 , Luhman,1990), Es Luhman (1990), quien postula que el campo educativo, viene a ser una especie de subsistema del sistema más general que es la sociedad, tomando esa afirmación, se puede predecir que la autonomía del sistema educativo esta signada por el sistema político, cultural y del fenómeno del poder. Panorama por el cual al escoger una carrera universitaria la elección gira en torno a las oportunidades laborales y no en la esencia propia de la educación superior, la cual tiene como génesis el diálogo de las ideas, los fundamentos del pensamiento y donde se buscaba dar respuesta a las preguntas ontológicas del ser humano.

La universidad fue institucionalizada en el medioevo, nace inmersa en el aparato político, su etimología tiene su origen en la siguiente explicación:

\footnotetext{
Universitas es un término latono derivado de unus, la unidad, y de verto que conlleva el sentido de volver. Conjugados estos elementos semánticos, universitas significó la unidad de personas congregadas, por ejemplo, en un gremio social, a veces conocido, en épocas posteriores al latín clásico, con diversos matices, como corpus, collegium, communio, societas o consortium, sin que en ninguno de estos casos la unidad significara uniformidad (Borrero, 2008, s/f).
}

Es a partir del siglo XII, con el incremento en el número de profesores y estudiantes que se establecen las primeras comunidades de profesores, con el propósito de defender sus intereses y la disciplina de sus estudios. Estas asociaciones ó universidades tenían por objeto la defensa de los profesores contra figuras como el canciller, el obispo, el rey o cualquiera que intentase ejercer sobre ellos un excesivo dominio. De modo semejante, los estudiantes se reunieron muy a menudo en corporaciones o ligas para protegerse contra los profesores, las autoridades municipales, entre otras. (Abbagnano y Visalberghi, 1992).

De esta manera, fue como paulatinamente el término universidad comenzó a ser asociado a las universidades de profesores y estudiantes, hecho con el que se pasa de la escuela catedralicia a la universidad como institución autónoma, se debe acotar que este el desarrollo de este proceso se produjo de manera gradual, y se cumplió de manera diferente para cada universidad en distintas épocas. (Abbagnano y Visalberghi, 1992).

En los albores del siglo XIX, "los movimientos reformistas de la educación apoyaron el aprendizaje basado en la pregunta o la investigación, trasladando el centro del aprendizaje de la memorización de conceptos y hechos a la búsqueda por parte de los estudiantes de respuestas a preguntas" (Villardón, 2015, p.42). Gracias a esta tendencia se fortalecen los enfoques de la educación superior en la que el aprendizaje se centra en el estudiante y en la necesidad de mejorar los vínculos entre la enseñanza y la investigación.

De esta manera, tiene sus inicios la academia, pero estos y otros paradigmas han permeado a lo largo de la historia en los métodos, objetos, sujetos y propósitos de la enseñanza, el debate sobre la veracidad de las ciencias aún genera discusiones álgidas dentro de los círculos de intelectuales. Para la segunda mitad del siglo XX surge una de las revoluciones silenciosas más profundas de la historia, se introdujo en las costumbres, las formas de producción, las relaciones políticas y en las mentalidades, y por supuesto en el sistema educativo (Proyecto agenda Uruguay, 2002).

Se puede afirmar que en esta era, el conocimiento la nueva fuente de poder, como en la edad media tiene la misma significación que tuvo la propiedad de la tierra o la máquina durante la era industrial, en la actualidad el conocimiento tiene un papel preponderante en el desarrollo de las naciones, pues "resulta incierto el futuro de una nación que no haya adoptado las definiciones estratégicas que le permitan acceder, almacenar, comprender, y utilizar el conocimiento científico" (Proyecto agenda Uruguay, 2002, p.36).

Este paradigma se fue instalando en el aparato educativo, y las universidades comenzaron a ser vistas como un bien de consumo con el cual se puede conseguir y desarrollar cierto estatus, de esta manera, se inició la masificación de la educación en la cual se implanta una inversión de capital humano. Este escenario se propago por el globo, integrando en muchas instituciones de educación superior, lo que paradójicamente ha llevado a 
aceptar que la educación superior y la investigación son elementos cruciales de la economía del conocimiento (Geuna, 1999), se puede acotar que son muchos los estudios que se han dedicado a explorar la relación entre la docencia y la investigación (Neumann, 1992, Marsh y Hattie, 2002 y Muller, 2000).

En tal sentido, el presente estudio se encargara de abordar esta temática descrita, de la cual no ha escapado la realidad peruana en la denominada sociedad del conocimiento que emerge la educación representa un gran desafío, en el que se debate la formación de ciudadanos especializados para laborar en los diferentes sectores productivos, sobrevivir a la era del conocimiento a través del impulso desmedido hacia la investigación o virar a la formación de individuos con un aparato crítico, capaz de analizar, comprender, organizar la información compleja que le proporciona la realidad, que logren integrar los nuevos conocimientos a su herencia cultural.

\section{Revisión de literatura}

Como consecuencia directa de las políticas económicas y sociales del neoliberalismo y la globalización las universidades de América Latina han sido presionadas para mantener sus proyecciones dentro del predominio y centralidad en los proyectos de Estado. Un ejemplo palpable es la historia de la expansión universitaria en el Perú, Para Cuenca (2015) este periodo se inició:

Entre 1960 y 1980, la matrícula universitaria pasó de ser de 30.000 a 255.000 y en el promedio de esos 30 años, el $79 \%$ de la matrícula se concentró en la oferta pública. Sin embargo, no es sino a partir del año 2000 que el crecimiento de la matrícula universitaria despegó (p. 10).

Como explica Cuenca (2015) este cambio obedece a una política del Estado peruano, la cual en al año de 1996 decide liberalizar el mercado educativo. Lo que permitió que las universidades generaran ingresos lucrativos con el propósito de atraer la inversión privada a fin de que esta financiara la creciente demanda de servicios de educación superior. Esta medida cambio drásticamente el sistema universitario, Abugattas (citado por Cuenca 2015):

\footnotetext{
Sostuvo que este giro en la finalidad universitaria de construcción de conocimiento por su aproximación (casi exclusiva) a satisfacer las necesidades del mercado laboral produjo un desfase entre la universidad y la sociedad. No solo el equilibrio entre la demanda y las necesidades del mercado de trabajo se alteró, sino que la investigación quedó postergada en la formación universitaria (p. 11).
}

El primer factor que contribuyó a la masificación de la educación superior en Perú fue un crecimiento económico significativo, de alrededor del 5,5\% del PIB, durante la década de los noventa (Wells, Cuenca, Blanco, Ramírez and Aragón, 2017, p .11). Así mismo, durante el gobierno de Alberto Fujimori, se confirió un carácter lucrativo a la educación, con el decreto legislativo 882 de 1996, en la Ley de Promoción de la Inversión en la Educación, permitiendo establecer universidades como personas jurídicas, comerciales y/o jurídicas (Cuenca 2015), como se puede observar la liberalización de la educación superior en el Perú se basó en una serie de proyecciones presupuestarias y un conjunto particular de supuestos. En este sentido Ricardo y Reátegui (2016) proponen tres hipótesis que argumentan la decisión de liberalizar la oferta educativa universitaria:

La primera consistió en que con la participación privada se conseguiría ampliar la oferta y, por lo tanto, la cobertura universitaria. La segunda fue que, como consecuencia de la primera, el acceso a los estudios superiores se democratizaría; es decir, más jóvenes de todos los estratos económicos estudiarían en la universidad. Finalmente, se supuso que el mercado sería un regulador efectivo de la calidad de este servicio, en el que la competencia dejaría fuera a las malas universidades (p. 6).

Pero las proyecciones se equivocaron y gracias a la descapitalización generada por el incremento desmedido de la población universitaria y la reducción presupuestal, la corrupción imperante en la gestión de los recursos y la privatización de los bienes y los servicios públicos, generaron una crisis en el sistema de educación superior, que se ha visto agravada por la influencia de los organismos multilaterales en las políticas estatales, en la medida en que los implementadores subnacionales reproducen una lógica de clientelismo y patrimonialista (Cuenca, 2015).

De igual manera, la mercantilización se impuso como una tendencia en el acceso, la permanencia y la titulación del sistema universitario, a consecuencia de esta situación las universidades públicas optaron por el autofinanciamiento, y buscaron distintas alternativas que le permitieran mantenerse activas; a saber es a través 
de la elaboración de proyectos, de la elevación del costo por trámites administrativos y servicios, como el comedor y la atención médica, que se logra mantener la universidad pública, La defensa de la universidad derivó en una lucha por buscar mejores rentas presupuestales ( Cuenca, 2015).

Este panorama, ocasionó el incremento de la desigualdad social, al igual que un paulatino desprecio por la diversidad cultural, y una persistente exclusión de los grupos étnicos minoritarios, además de una distribución presupuestaria dispareja que emerge de una política centralista, en la cual la capital es el centro de todos los poderes (Cuevas, 2015). A su vez, se originó un filtro para el acceso a la educación superior, según el estudio de Sánchez y Singh (2018), se demuestra que, en países como la India y Perú, la selectividad en educación superior opera a nivel de la institución caracterizada por un estricto requerimiento para la admisión a las universidades de élite, ya sea a través de exámenes o pruebas específicas de las instituciones. Sin embargo, una amplia variedad instituciones de educación superior ha condicionado su ingreso a la capacidad de pago.

Las universidades en este caso fueron vistas como parte de un sistema que se utiliza como recurso para la materialización efectiva de la generación del conocimiento rentable a las actividades económicas del país a fin de obtener prestigio a través del salario de sus egresados y del número de publicaciones sin tener en consideración la carrera docente (Pineda y Celis, 2017).

Por tanto, la situación antes expuesta ha conllevado, a que la investigación científica sea muy incipiente, pues con la universidades publicas desarrollando otro tipo de actividades más lucrativas que le generan mayores ingresos, es muy poco el presupuesto que se asigna al fomento y desarrollo de la investigación científica, o el poco presupuesto asignado a la investigación es repartido en los docentes investigadores a manera de incentivo, por otra parte los docentes no son investigadores activos, y es a través de la publicación de artículos científicos el medio idóneo para la difusión de la ciencia, al no poseer investigaciones no hay trabajos que difundir.

\section{Hacia la educación que queremos}

Como ya se ha argumentado, la investigación científica es un factor importante en el desarrollo social y económico de un país, es uno de los principales elementos que inciden en la capacidad para mejorar las condiciones de vida de las sociedades, es el recinto universitario uno de los lugares predilectos para fomentar y desarrollar la investigación, incluyendo dentro de sus diseños curriculares, contenidos programáticos a fin de que sea la nueva generación la que contribuya con el desarrollo sostenible del país.

No obstante, en el campo de la investigación, se han instaurado firmemente métodos de evaluación de la productividad e impacto tanto para académicos individuales, como para instituciones. De modo indirecto, en ambos casos se introducen procedimientos de tasación (monetarios y de prestigio) para el desempeño individual e institucional. (Henríquez 2018, p. 58).

Con referencia a la situación de la educación universitaria en Perú, Cuevas (2015) agrega que para que la educación universitaria pueda constituirse como un instrumento que aporte para el desarrollo del país se debe remediar ciertos asuntos, aboga por el uso adecuado de la nueva ley universitaria y por la puesta en marcha de proyectos y otras acciones que se encaminen a mejorar la situación ya descrita.

En tal sentido, la ley universitaria 30220 (Diario oficial El Peruano, 2014) fue aprobada en el Congreso de la República, el 26 de junio del 2014, y promulgada el 8 de julio por el presidente Ollanta Humala, esta extensa ley contiene 133 artículos y englobe tres dimensiones del sistema universitario, en esta se crea además, la Superintendencia Nacional de Educación Superior Universitaria - SUNEDU, con la finalidad de supervisar la calidad del servicio educativo universitario y de fiscalizar si los recursos públicos y los beneficios otorgados por el marco legal a las universidades, han sido destinados a fines educativos y al mejoramiento de la calidad del servicio.

Otro punto importante para el principio del cambio, se describe en el Informe anual emitido por SUNEDU (2017) sobre el uso de beneficios otorgados a universidades privadas, en el artículo 118 de la Ley Universitaria concerniente a la promoción de la inversión privada en educación, se informa que la reinversión de utilidades o excedentes de las universidades privadas se aplica en infraestructura, equipamiento para fines educativos, 
investigación e innovación en ciencia y tecnología, capacitación y actualización de docentes, proyección social, apoyo al deporte de alta calificación y programas deportivos; así como la concesión de becas.

Aunado a este plan de fortalecimiento de la investigación, se encuentra CONCYTEC, una institución que cumple un rol importante, ya que se encarga de estudiar el desarrollo de la investigación científica en el país, cuál es su influencia en la calidad de la universidad, el impacto de la presencia de la investigación en la universidad en la calidad profesional y el salario de los egresados, en la modalidad de la educación técnica, se observan los estándares para evaluarla, investigar cuál es el déficit de técnicos, la demanda de técnicos en los diferentes sectores económicos, a fin de garantizar una adecuada inserción de estos profesionales al mercado laboral (Ñopo y Kitmang, 2017).

Sin embargo, la realidad del docente es otra, según el informe bienal sobre la realidad universitaria (2018), para el año 2015 existían 84.774 puestos docentes universitarios, de esta suma el 10,5\% es ocupado por docentes con grado de doctor, el $27,4 \%$ con grado de maestría, el $59,5 \%$ con grado de bachiller y un 2,6\% sin grado. Cabe destacar, que para el año 2015 aún prevalecía una brecha para cumplir con lo establecido por el artículo 82 de la Ley $N^{\circ} 30220$, (Diario oficial El Peruano, 2014) en la que se reglamentan los grados académicos mínimos para el ejercicio de la docencia en sus diferentes niveles y para lo cual se ha establecido una meta de cumplimiento gradual a cinco años, en otras palabras, en el año corriente los docentes universitarios deben tener títulos de magíster y doctorado para poder ejercer la docencia en las instituciones de educación superior.

De igual manera, al analizar los artículos de la ya referida Ley Universitaria $\mathrm{N}^{\circ} 30220$, (Diario oficial El Peruano, 2014) referentes a la carrera docente, en el capítulo VIII, artículo 79 se indica que las dentro de las funciones de los docentes se encuentran : "la investigación, el mejoramiento continuo y permanente de la enseñanza, la proyección social y la gestión universitaria, en los ámbitos que les corresponde", sin embargo es en artículo 86, en el cual se define al docente investigador, "es aquel que se dedica a la generación de conocimiento e innovación, a través de la investigación. Es designado en razón de su excelencia académica". Asimismo, la ley indica que dicho docente recibe una bonificación especial del cincuenta por ciento $(50 \%)$ de sus haberes totales. Y es el Vicerrectorado de Investigación o la autoridad competente que evalúa cada dos años, la producción de los docentes, para su permanencia como investigador; en el marco de los estándares del Sistema Nacional de Ciencia, Tecnología e Innovación Tecnológica (SINACYT).

Todo este contexto, apuntala a catapultar la universidad como un organismo en el que se desarrollen estudios innovadores encaminados al crecimiento económico y social de la nación, por ende, se están realizando cambios en las políticas educativas a fin de lograr esta meta a través de la divulgación de la producción de la investigación científica, para llegar a esta meta se hace necesario adoptar medidas que fomenten el desarrollo de la ciencia, por tanto, este estudio propone analizar el impacto de estas directrices en el sistema de educación superior.

\section{Metodología}

La investigación se planteó como un estudio documental en el que se recopiló información de artículos publicados en revistas científicas indexadas en bases de datos de impacto mundial y regional Scimago Journal \& Country Rank e informes y reportes oficiales de organismos nacionales como SUNEDU.

Se revisaron 1 ley, 7 artículos científicos, 3 informes y/o reportes oficiales y 12 fuentes documentales, para un total de 23 documentos.

El procesamiento de la información se realizó mediante tablas, gráficos y un análisis estadístico descriptivo de la información recopilada, los datos de interés giran en torno al impacto que presentan las publicaciones de los artículos científicos a través del número de citas, distribución de documentos, documentos citados, citas y autocitas, distribución de citas de países por documento e índice de H para el año 2017, distribución de documentos publicados entre los años 2010 al 2017, número de revistas indexadas en SCOPUS entre los años 2010 al 2017, además de las tablas elaboradas a partir del nuevo Reglamento de calificación, clasificación y registro de los investigadores del SINACYT. 
Luego del estudio cuantitativo de los datos, se realizó el análisis y la discusión cualitativa de la información, en donde, a través del análisis crítico y reflexivo, se concatenaron ideas de diferentes autores con los resultados observados, llegando a conclusiones y recomendaciones inherentes al tema.

\section{Resultados}

Atendiendo a las estadísticas de Scimago Journal \& Country Rank que trabaja con datos de Scopus, Perú ha incrementado su participación en la ciencia mundial considerando el número de artículos científicos publicados por sus investigadores; Distribución de Documentos, documentos citados, citas y autocitas año 2017, como se puede apreciar a continuación en el gráfico 1.

Gráfico 1 - Distribución de Documentos, documentos citados, citas y autocitas, año 2017

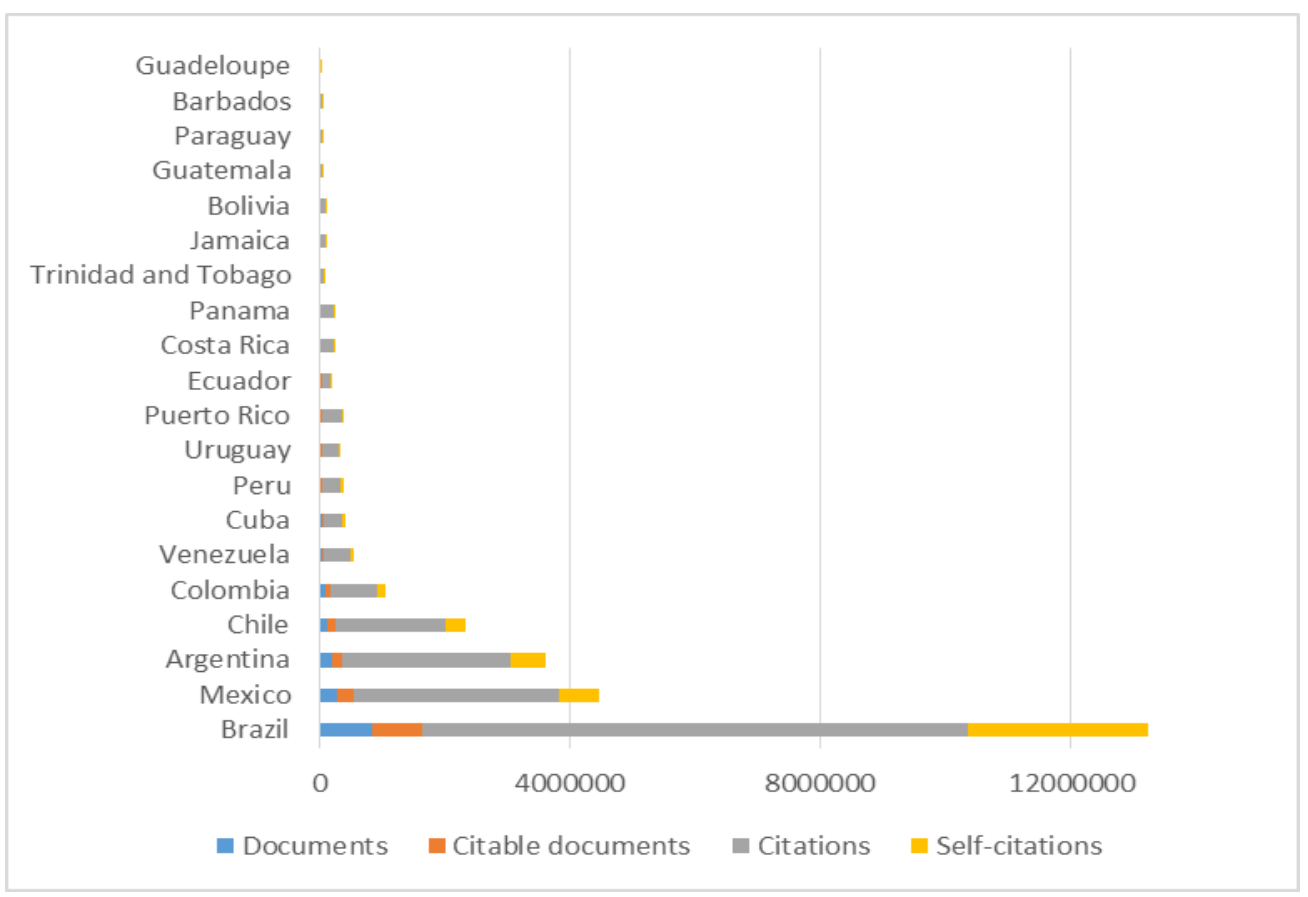

Fuente: Elaboración propia a partir de datos de Scimago Journal \& Country Rank

Como se puede apreciar en el gráfico 1, para el año 2017 Perú se encuentra en el puesto número 8 dentro de los primeros 20 países en el ranking de Scimago Journal \& Country Rank, con un total de 20308 documentos, 18467 documentos citados, 304057 citas y 32178 autocitas, pero aún se encuentra por debajo de países como Cuba y Venezuela que presentan situaciones políticas y sociales extremas, se ha apreciado notables mejoras en el caso peruano, pero aún se encuentra por debajo del nivel de los países de América Latina. 
Gráfico 2 - Distribución de Citas de países por documento e índice de H para el año 2017

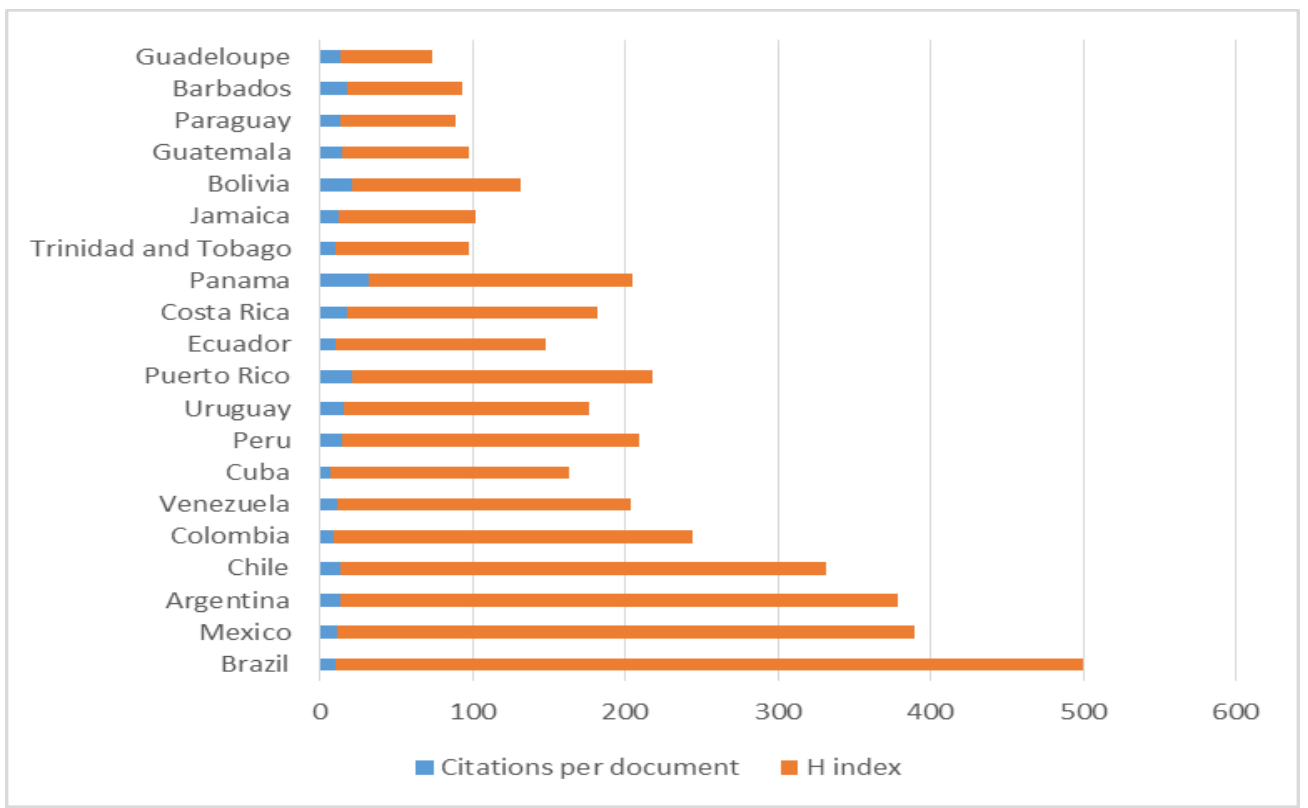

Fuente: Elaboración propia a partir de datos de Scimago Journal \& Country Rank

Se puede apreciar en el gráfico 2, que Perú se obtuvo un 14,97 sobre citas por documento entre los 20 primeros países, con un índice $\mathrm{H}$ igual a 194, que lo deja por debajo de países como Brasil con 489, México 378, Argentina 364, Chile 318 y Colombia que lo sobrepasa con un índice $\mathrm{H}$ de 235 , lo que indica que estos países han recibido mayor citación en sus publicaciones.

Gráfico 3 - Distribución de documentos publicados entre los años 2010 al 2017

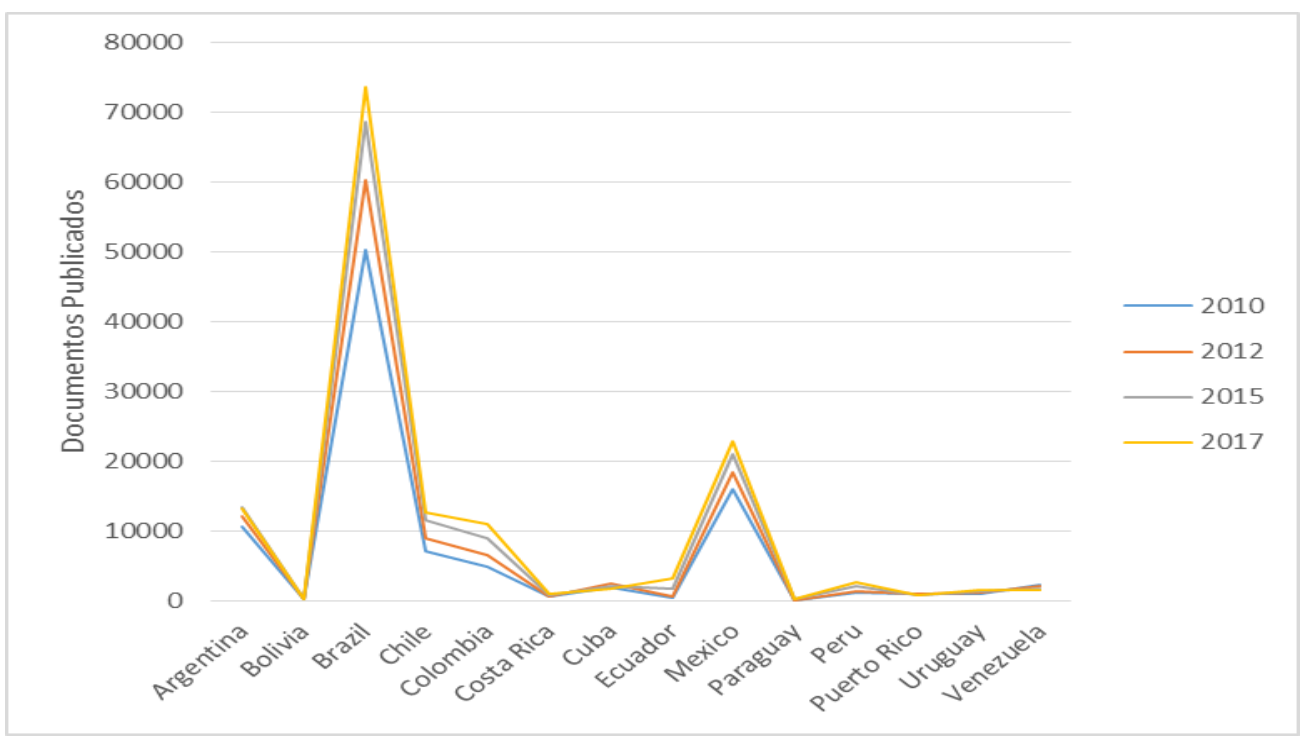

Fuente: Elaboración propia a partir de datos de Scimago Journal \& Country Rank

Como se observa en la Figura 3, se puede constatar que la actividad de investigación científica en el país ha crecido, se están produciendo documentos y así lo demuestra la distribución de documentos publicados entre 
los años 2010 al 2017. En el año 2010 solo se alcanzó a publicar un total de 1085 documentos, para el año 2012 se publicaron 1374, así mismo en el 2015 se alcanzarían 2027 publicaciones y para el año 2017 se publicaron 2597, efectivamente se denota una tendencia al incremento, además de ser relativamente lento, lo que ocasiona que aún se encuentre por debajo de otros países de América Latina.

Gráfico 4 - Número de revistas indexadas en SCOPUS entre los años 2010 al 2017

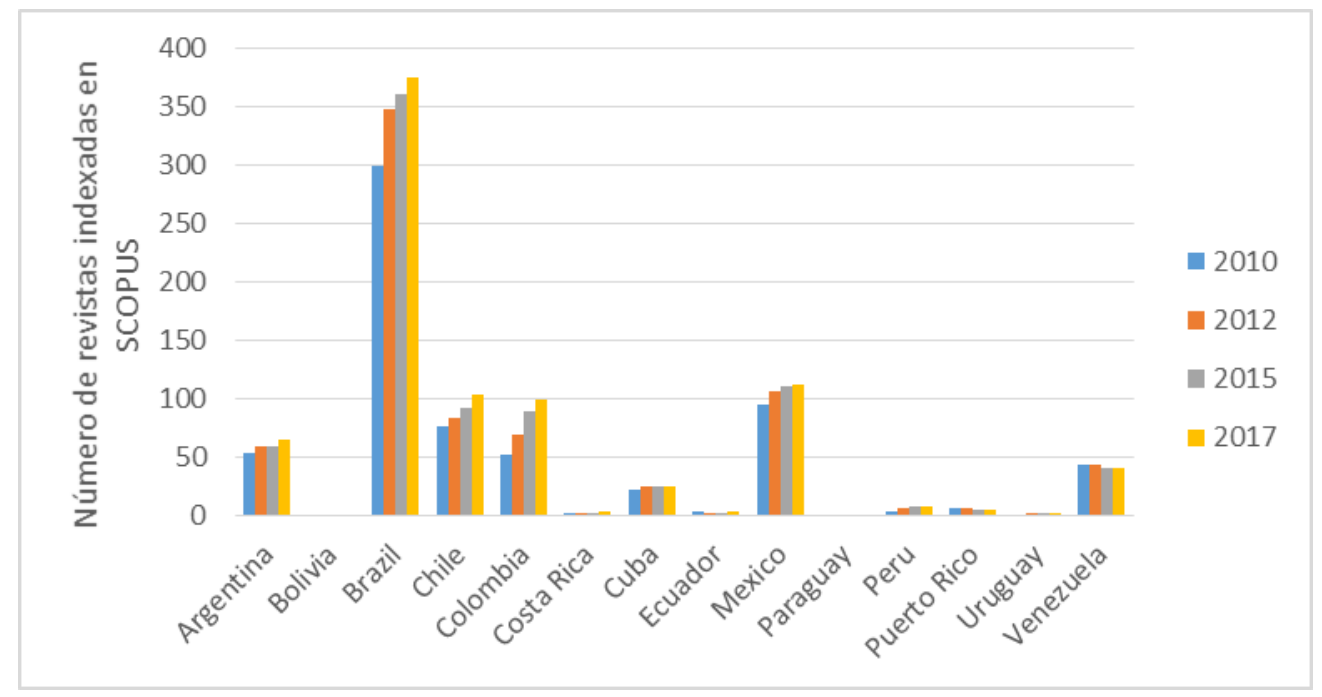

Fuente: Elaboración propia a partir de datos de Scimago Journal \& Country Rank

En la Figura 4 se observa el número de revistas indexadas en SCOPUS entre los años de 2010 al 2017, en este caso Perú aumentó entre 2010 y 2017 de 3 a 7, crecimiento que sin embargo no ha significado una mejora posicional respecto al resto de países de la región, para el año 2010 se contaba con 3 revista, en el 2012 se duplica el monto con 6 revistas, para el año 2015 suben a 7 el número de revistas y así se mantiene hasta el 2017,el fenómeno obedece a que sus publicaciones se elevan internamente pero no en alto impacto, al contrario de países como Brasil que publica investigaciones de nivel, con un alto impacto y además recibe publicaciones de otros países posicionándole en el primer lugar.

En cuanto a la siguiente fuente de información, se tiene al Reglamento de calificación, clasificación y registro de los investigadores del sistema nacional de ciencia, tecnología e innovación tecnológica (Reglamento RENACY), del cual se han elaborado unas tablas a fin de visualizar los criterios que se están planteando para la clasificación de los investigadores.

Tabla 1. Criterios de evaluación para la clasificación como investigador del grupo "María Rostworowski” en el RENACYT.

\begin{tabular}{|l|l|l|l|}
\hline \multicolumn{1}{|c|}{ Criterio/Nivel } & Nivel I & Nivel II & Nivel III \\
\hline A. Tener grado de "X" reconocido por SUNEDU. 1 & $\begin{array}{l}\text { Doctor o } \\
\text { maestro }\end{array}$ & $\begin{array}{c}\text { Doctor o } \\
\text { maestro }\end{array}$ & $\begin{array}{c}\text { El grado de bachiller } \\
\text { y el título profesional }\end{array}$ \\
\hline $\begin{array}{l}\text { B. Tener como mínimo "X" artículos científicos en revistas } \\
\text { indexadas en base de datos que cumplan con un proceso de } \\
\text { revisión de pares externos y otros estándares internacionales.2, } \\
3,4\end{array}$ & $X>10$ & $X>6$ & $X>3$ \\
\hline
\end{tabular}




\begin{tabular}{|c|c|c|c|}
\hline Criterio/Nivel & Nivel I & Nivel II & Nivel III \\
\hline $\begin{array}{l}\text { C. Tener "X" publicaciones de libros y/o capítulos de libros en su } \\
\text { especialidad indexadas en base de datos que cumplan con un } \\
\text { proceso de revisión de pares externos y otros estándares } \\
\text { internacionales2, 3; y/o tener registros de propiedad intelectual } \\
\text { (como patentes u otras modalidades de protección de } \\
\text { invenciones o nuevas tecnologías) concedidas y registradas en } \\
\text { INDECOPI. }\end{array}$ & $x \geq 3$ & $x \geq 2$ & $x \geq 1$ \\
\hline $\begin{array}{l}\text { D. Haber participado como mínimo en "X" proyectos de } \\
\text { investigación (en "Y" de ellos debe haber participado como } \\
\text { investigador principal, se incluye su proyecto de doctorado y } \\
\text { posdoctorado). } 4,5,6\end{array}$ & $\begin{array}{l}X \geq 25 \\
Y \geq 1\end{array}$ & $x \geq 15$ & $x \geq 1$ \\
\hline $\begin{array}{l}\text { E. De ser el caso haber participado en "X" proyectos de } \\
\text { investigación para la empresa. El documento de sustentación } \\
\text { será proporcionado por el solicitante. } 6\end{array}$ & $x \geq 0$ & $X \geq 0$ & $x \geq 0$ \\
\hline $\begin{array}{l}\text { F. Haber asesorado o co-asesorado como mínimo " } X \text { " tesis } \\
\text { sustentadas entre ellas de posgrado y/o pregrado. } 7\end{array}$ & $x \geq 4$ & $x \geq 2$ & ----- \\
\hline
\end{tabular}

Tabla 2. Criterios de evaluación para la clasificación como investigador del grupo "Carlos Monge Medrano" en el RENACYT.

\begin{tabular}{|c|c|c|c|c|}
\hline Criterio/Nivel & Nivel I & Nivel II & Nivel III & Nivel IV \\
\hline A. Tener grado de "X" reconocido por SUNEDU. 1 & $X=$ Doctor & $X=$ Doctor & $X=$ Doctor & $\begin{array}{c}\text { X = Doctor, en } \\
\text { los últimos } 7 \\
\text { años }\end{array}$ \\
\hline $\begin{array}{l}\text { B. Tener como mínimo " } X \text { " artículos científicos en } \\
\text { revistas indexadas en base de datos que cumplan con } \\
\text { un proceso de revisión de pares externos y otros } \\
\text { estándares internacionales2, 3, reconocidas por la } \\
\text { DEGC del CONCYTEC. }\end{array}$ & $\begin{array}{l}X \geq 40 \text { (un } \\
50 \% \text { de los } X \\
\text { artículos debe } \\
\text { estar en } Q 2 \text { ) }\end{array}$ & $\begin{array}{l}X \geq 20 \text { (un } \\
10 \% \text { de los } \\
X \text { artículos } \\
\text { debe estar } \\
\text { en } Q 4 \text { ) }\end{array}$ & $x \geq 9$ & $x \geq 3$ \\
\hline $\begin{array}{l}\text { C. Tener "X" publicaciones de libros y/o capítulos de } \\
\text { libros en su especialidad indexadas en base de datos } \\
\text { que cumplan con un proceso de revisión de pares } \\
\text { externos y otros estándares internacionales2, 3, } \\
\text { reconocidas por la DEGC del CONCYTEC. } \\
\text { Así mismo, tener registros de propiedad intelectual } \\
\text { (como patentes u otras modalidades de protección de } \\
\text { invenciones o nuevas tecnologías) concedidas y } \\
\text { registradas en INDECOPI o SCOPUS. }\end{array}$ & $x>2$ & $x>1$ & $x \geq 1$ & ---- \\
\hline $\begin{array}{l}\text { D. Haber participado como mínimo en "X" proyectos de } \\
\text { investigación (en "Y" de ellos debe haber participado } \\
\text { como investigador principal, se incluye su proyecto de } \\
\text { doctorado y posdoctorado). }\end{array}$ & $\begin{array}{l}X \geq 12 \\
Y=5\end{array}$ & $\begin{array}{l}X \geq 8 \\
Y=3\end{array}$ & $\begin{array}{l}X \geq 5 \\
Y=1\end{array}$ & $\begin{array}{l}X \geq 1 \\
Y=1\end{array}$ \\
\hline $\begin{array}{l}\text { E. De ser el caso haber participado en "X" proyectos de } \\
\text { investigación para la empresa. El documento de } \\
\text { sustentación será proporcionado por el solicitante. } 6\end{array}$ & $x \geq 0$ & $x \geq 0$ & $x \geq 0$ & $x \geq 0$ \\
\hline $\begin{array}{l}\text { F. Líder de grupo de investigación o laboratorio de } \\
\text { investigación en CTI o haber asesorado o co-asesorado } \\
\text { como mínimo "X" tesis sustentadas entre ellas "Y" de } \\
\text { posgrado8. }\end{array}$ & $\begin{array}{l}X \geq 5 \\
Y \geq 2\end{array}$ & $\begin{array}{l}X \geq 3 \\
Y \geq 0\end{array}$ & $x \geq 1$ & ---- \\
\hline
\end{tabular}


En las tablas se puede observar como se establece el acceso a la calificación como investigador, teniendo en cuenta que los puntos $\mathrm{A}$ y $\mathrm{B}$ son obligatorios (incluye equivalencias) y de los puntos de $\mathrm{C}$ a $\mathrm{F}$ se deberá cumplir con tres criterios como mínimo, estos lineamientos demuestran la intención de elevar el nivel de los investigadores, ya que para ser calificados deben presentar una verdadera producción científica, no solo con la publicación de artículos científicos sino también con la elaboración de libros, participación en proyectos de investigación, liderazgo de grupo de investigación o laboratorios de investigación y asesoría de tesis sustentadas entre ellas pregrado y posgrado.

\section{Conclusiones}

El estudio ha demostrado que existe una disposición por elevar el nivel de la producción científica en el país, pero aún no se ha logrado obtener resultados óptimos, cabe recalcar, que si se quiere ser reconocido como investigador dentro de la comunidad científica internacional, se debe tener una amplia lista de artículos en publicaciones científicas disponibles en revistas indizadas en bases de datos internacionales como por ejemplo: ISI/WoK, Medline/PubMed, Scopus, es absurdo denominarse investigador sin tener publicaciones, pues se estaría definiendo como un teórico de la investigación, pero que no tiene la experiencia en la elaboración de proyectos de investigación que den lugar a la publicación de un artículo científico o una patente, teniendo en consideración que esto desemboca en un nuevo conocimiento que beneficia de manera directa a la sociedad en la que se desarrolla la investigación.

Para lograr este fin se ha diseñado el Reglamento de calificación, clasificación y registro de los investigadores del sistema nacional de ciencia, tecnología e innovación tecnológica o Reglamento RENACYT, que para cumplir los parámetros impuestos por tal, se debe considerar lo siguiente: En primer lugar, para publicar en revistas de alto impacto se requiere cierto perfil, en principio se debe poseer una investigación innovadora e inédita que derive en un artículo científico de calidad, que siga todos los parámetros exigidos por la revista. No es un secreto que el porcentaje de aceptación en revista de alto impacto es muy bajo; ya que no sólo se toma en cuenta el artículo presentado, además se estudia el perfil del investigador, la cantidad de sus artículos publicados y la particularidad de sus investigaciones, no es tarea fácil para un investigador novel publicar en revistas indexadas.

En segundo lugar; como se puede ratificar, publicar artículos científicos en revistas de alto impacto es un requerimiento básico para un investigador, en cuanto a este aspecto como se mencionó en el capítulo anterior, se ha diseñado el Reglamento de calificación, clasificación y registro de los investigadores del sistema nacional de ciencia, tecnología e innovación tecnológica o Reglamento RENACYT, en el cual se establece el perfil del investigador, existen criterios de evaluación para la clasificación como investigador en los dos grupos: María Rostworowski y Carlos Monge Medrano, si se analizan las bases de este estatuto se debe tomar en cuenta que:

- Los artículos científicos deben ser publicados en revistas indexadas que cumplan con un proceso de revisión de pares externos y otros estándares internacionales reconocidos por la DEGC del CONCYTEC.

- Para el nivel I de María Rostworowski:

- Los artículos publicados en SCOPUS equivalen a su vez a artículos publicados en: Web of Science, Wiley, IEEE Xplore, Mathematical Reviews, Medline, SPIE digital Library, Springer, McGraw-Hill.

- 10 Artículos SCOPUS equivalen a 20 artículos publicados en Taylor \& Francis Group, EconLit, ProQuest, Bentham Science.

- 10 artículos SCOPUS equivalen a 30 artículos publicados en Scielo, DAOJ, Redalyc o Latindex.

- Para Nivel II de María Rostworowski

- Se reglamentan 6 artículos SCOPUS o su equivalente o Web of Science, Wiley, IEEE Xplore, Mathematical Reviews, Medline, SPIE digital Library, Springer, McGraw-Hill o 12 artículos publicados en Taylor \& Francis Group, EconLit, ProQuest, Bentham Science o a su vez a 18 artículos publicados en Scielo, DAOJ, Redalyc o Latindex.

- Para el Nivel III de María Rostworowski 
- 3 SCOPUS o su equivalente en Web of Science, Wiley, IEEE Xplore,

Mathematical Reviews, Medline, SPIE digital Library, Springer, McGraw-Hill, o 6 artículos publicados en Taylor \& Francis Group, EconLit, ProQuest, Bentham Science o 9 artículos publicados en plataformas Scielo, DAOJ, Redalyc o Latindex.

En cuanto a los criterios propuestos en la tabla 2 para la clasificación como investigador del grupo "Carlos Monge Medrano" en el RENACYT, se puede destacar que:

- Para el nivel I de Carlos Monge Medrano

- Se requieren 40 SCOPUS o su equivalente Web of Science, Wiley, IEEE Xplore, Mathematical Reviews, Medline, SPIE digital Library, Springer, McGraw-Hill, de los cuales el $50 \%$, es decir 20 deben ser Q2. O su equivalente que serían 80 artículos publicados en Taylor \& Francis Group, EconLit, ProQuest, Bentham Science, o 120 artículos publicados en Scielo, DAOJ, Redalyc o Latindex.

- Para el nivel II de Carlos Monge Medrano

- Se solicitan 20 SCOPUS o su equivalente Web of Science, Wiley, IEEE Xplore, Mathematical Reviews, Medline, SPIE digital Library, Springer, McGraw-Hill, de los cuales $10 \%$, deben ser Q4. O su equivalente que seria 40 artículos publicados en Taylor \& Francis Group, EconLit, ProQuest, Bentham Science, o 60 artículos publicados en Scielo, DAOJ, Redalyc o Latindex.

- Para el Nivel III de Carlos Monge Medrano

- Se reglamentan 9 artículos SCOPUS o su equivalente Web of Science, Wiley, IEEE Xplore, Mathematical Reviews, Medline, SPIE digital Library, Springer, McGraw-Hill, o 18 artículos publicados en Taylor \& Francis Group, EconLit, ProQuest, Bentham Science, o su equivalente 27 Scielo, DAOJ, Redalyc o Latindex.

- Para el nivel IV de Carlos Monge Medrano

- Se requieren 3 artículos SCOPUS o su equivalente Web of Science, Wiley, IEEE Xplore, Mathematical Reviews, Medline, SPIE digital Library, Springer, McGraw-Hill, o su equivalente 6 artículos publicados en Taylor \& Francis Group, EconLit, ProQuest, Bentham Science o 9 artículos publicados en Scielo, DAOJ, Redalyc o Latindex.

Como premisa se debe tener en consideración que el país está abogando por un cambio que potencie la producción científica, y este incide directamente en el papel que juega la universidad, y sobre todo de los docentes quienes son los encargados de producir, fomentar y enseñar las tareas propias de la investigación, por ende se aboga por transformar el enfoque de graduar ciudadanos, sino también por prepararlos para que realicen aportes significativos al país, además se lucha por elevar el nivel de los docentes, en tal sentido Zabalza (2004) asevera:

Se está pidiendo a las universidades que no se contenten con trasmitir la ciencia sino que deben crearla (esto es, deben combinar la docencia y la investigación), que den un sentido práctico y profesionalizador a la formación que ofrecen a los estudiantes, y que hagan todo eso sin cerrarse sobre sí mismas sino en contacto con el entorno social, económico y profesional en cuya mejora deben colaborar (p. 22).

En tal sentido, el rango que deben cumplir los investigadores, y los docentes que deseen se clasificados como investigadores es elevado y será una ardua labor obtener el perfil en poco tiempo, pues si toma en consideración que para la publicación de un artículo el lapso de espera varia de 6 meses a un año, dependiendo de la periodicidad de la revista, pero según este reglamento un investigador deberá publicar un margen de 15 artículos anuales para poder cumplir con los preceptos de los últimos niveles, sin tomar en consideración el listado de obras de producción intelectual y científica que están obligados a ostentar.

De igual manera, ante tal situación, los investigadores se verán obligados a publicar en el extranjero, lo cual tendrá un impacto negativo en las publicaciones de revistas nacionales, en primer lugar si no se ayuda a la 
investigación nacional no se elevará el nivel de las revistas y en segundo lugar al no tener publicaciones las revistas tenderán a desaparecer.

En cuanto al docente universitário, cabe recalcar que debe reunir dentro de su perfil un conjunto de características en las que se mezclan; sus creencias, intereses, cosmovisión, su motivación, su responsabilidad, empatía, respeto a su institución y a sus estudiantes, estos elementos definen su profesionalismo y la capacidad que posee para desempeñar su labor, pero sus objetivos y finalidades obedecen a políticas emanadas por el Estado en el caso de las instituciones públicas.

Como conclusión del estudio, se debe ponderar la intención del Estado al propiciar políticas que se encaminan a desarrollar la investigación científica, sin embargo, para ello los académicos deben contar con el visto bueno de las instituciones académicas a fin crear un plan de colaboración que permita mejorar la praxis docente e incentivar la investigación científica. Por lo cual, Marina (2007) afirma que: "la investigación y la aplicación de sus resultados se vinculan a la universidad como función básica de ésta, además son sus funciones el enriquecimiento intelectual, moral y material de la sociedad" (p. 69).

En tal sentido, ante esta expectativa los retos que presenta la investigación en educación superior son de gran envergadura, se debe abogar por una formación docente que se encargue de potenciar el espíritu del investigador a fin de que los docentes puedan elevar su nivel al perfil requerido por el nuevo reglamento de clasificación, y por ende teniendo una mejor formación los docentes reconocerán el valor de la investigación y podrán formar estudiantes inculcando y desarrollando las tareas propias de la investigación.

\section{Bibliografía}

Abbagnano, N. y Visalberghi A. (1992). Historia de la pedagogía. España: Fondo de Cultura Económica.

Borrero, C. A. (2008). La universidad. Estudios sobre sus orígenes, dinámicas y tendencias. Bogotá: Editorial Pontificia Universidad Javeriana.

Cuenca, R. (Ed.). (2015). La educación universitaria en el Perú. Democracia, expansión y desigualdades. Lima: IEP. Instituto de estudios peruanos.

Cuenca, R. y Reátegui, L (2016). La (incumplida) promesa universitaria en el Perú. Lima: IEP.

Geuna, A. (1999). The Economics of Knowledge Production. Funding and the Structure of University Research. Cheltenham: Edward Elgar.

Henríquez, P. (Ed.) (2018). El papel estratégico de la educación superior en el desarrollo sostenible de América Latina y EI Caribe. Argentina: UNESCO.

Ibarra, E. (2003). Capitalismo académico y globalización: la universidad reinventada. Educação \& Sociedade, 24(84), 10591067. http;//doi.org/10.1590/S0101-73302003000300017.

Luhmann, N. (1990). Sociedad y sistemas: la ambición de la teoría. Barcelona: ediciones Paidós / I.C.E- U.A.B.

Marina, T. (Ed.). (2007). Reconstruir la universidad a través del cambio cultural. España: Universitat Autónoma de Barcelona.

Marsh, H.W. y Hattie, J. (2002). The relation between research productivity and teaching effectiveness. The Journal of Higher Education, 73, pp. 601-641. http://doi.org/10.3102/00346543066004507.

Maturana, H. y Varela, F. (1994). De máquinas y seres vivos. Autopoiesis: la organización de lo vivo. Santiago de Chile: Editorial Universitaria.

Muller, C.A. (2000). Linking research and teaching: a study of graduate student engagement. Teaching in Higher Education, 5 , pp. 5-21. http://doi.org/10.1080/135625100114920.

Neumann, R. (1992). Perception of the teaching-research nexus: A framework for analysis. Higher Education, Vol. 23, No. 2, Cumulative Index of Higher Education 1972-1990 (Mar., 1992), pp. 159-171. [citado 2019-10-02] Disponible en: https://www.jstor.org/stable/3447303.

Ñopo, H. y Kitmang, J. (2017) Balance de investigación en políticas públicas 2011 - 2016 y agenda de investigación 2017 2021. Lima: CIES.

Pineda, P., y Celis, J. (2017). ¿Hacia la Universidad Corporativa? Reformas Basadas en el Mercado e Isomorfismo Institucional en Colombia. Education Policy Analysis Archives, 25(71). http://doi.org/10.14507/epaa.25.283. 
Proyecto agenda Uruguay. (2002). Educación para la sociedad del conocimiento. Aportes hacía una política de Estado. Uruguay: Ediciones Trilce.

Ley Universitaria 30220, 9 de julio de 2014. Diario Oficial El Peruano (2014). [citado 2019-10-02] Disponible en: http://www.leyes.congreso.gob.pe/Documentos/Leves/Textos/30220.pdf.

Sánchez, A. \& Singh, A. (2018). "Accessing higher education in developing countries: Panel data analysis from India, Peru, and Vietnam," World Development, Elsevier, vol. 109(C), pages 261-278. http://doi.org/10.1016/j.worlddev.2018.04.015.

SUNEDU. (2017). Informe anual. De uso de beneficios otorgados a universidades privadas- 2015. [citado 2019-10-02] Disponible en: https://www.sunedu.gob.pe/informe-uso-beneficios-otorgados-universidades-privadas/

SUNEDU. (2018). Informe bienal sobre la realidad universitaria peruana. [citado 2019-10-02] Disponible en: https://www.sunedu.gob.pe/informe-bienal-sobre-realidad-universitaria/

Villardón Gallego, L. (2015). Competencias genéricas en educación superior. Metodologías específicas para su desarrollo. Madrid: Narcea ediciones.

Wells, R. S.; Cuenca, R.; Blanco, G.; and Aragón, J. (2017) "Geographic mobility and social inequality among Peruvian university students". Higher Education. http://doi.org/10.1007/s10734-017-0149-6.

Zabalza, M. (2004). La enseñanza universitaria. El escenario y sus protagonistas. Madrid: Ediciones NarceaAuthor data 


\section{Datos de los autores}

Isral Barrutia Barreto

Doctor en Administración. Doctor en Investigación. Magister en Administración y Dirección de empresas.

Docente en la Universidad Científica del Sur, Perú.

ibarrutia@innovascientic.com

Erika Raquel Acosta Roa

Profesora en la Especialidad de Geografía e Historia, Magister en Educación Mención Enseñanza de la Historia, Doctora en Cultura y Arte para América Latina y El Caribe. Jefa de Artículos científicos Innova Scientific, Perú. innovasciencia@gmail.com

Manuel Quipuscoa Silvestre

Doctor en Ciencias de la Educación. Magister en Educación Mención en Pedagogía Universitaria. Coordinador Administrativo de PREFORD de Facultad de Educación en la Universidad Nacional de Trujillo, Perú.

mquipusil@gmail.com

\section{Herbert Victor Huaranga Rivera}

Doctor en Contabilidad. Doctor en Eduación. Magíster en Educación Mención: Docencia y Gestión Educativa. Docente en la Universidad Nacional Intercultural Selva Central Juan Santos Atahualpa, Perú.

hebhurisc@gmail.com

Received - Recibido: 2019-02-13

Accepted - Aceptado: 2019-07-22

\section{$(\mathrm{cc}) \mathrm{BY}$}

This work is licensed under a Creative Commons Attribution 4.0

United States License.

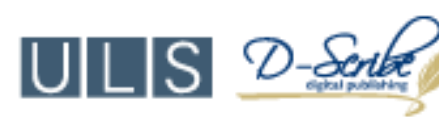

This journal is published by the University Library System of the University of Pittsburgh as part of its D-Scribe Digital Publishing Program and is cosponsored by the University of Pittsburgh Press. 